\title{
Relapsing polychondritis presenting 2 years after systemic sclerosis with pulmonary arterial hypertension
}

Li Su1,,\#, Na Zhang ${ }^{1, \#}$, Hui Wang ${ }^{1}$, Zhenwen Yang ${ }^{2, *}$, Wei Wei ${ }^{1, *}$

${ }^{1}$ Department of Rheumatology and Immunology, Tianjin Medical University General Hospital, Tianjin Medical University, Tianjin, China

${ }^{2}$ Department of Cardiology, Tianjin Medical University General Hospital, Tianjin Medical University, Tianjin, China

\begin{abstract}
Relapsing polychondritis (RPC) is a systemic immune-mediated disease characterized by recurrent and progressive inflammation of cartilaginous tissues. $64 \%$ of RPC patients concurrent with other autoimmune disorders, there are very few reports about the concomitant RPC patients with systemic sclerosis (SSc). Herein we report a case of RPC in a 50-yearold female following SSc with pulmonary arterial hypertension (PAH) 2 years ago. She was treated with corticosteroids, immunosuppressive drugs, oral endothelin-A receptor antagonist and phosphodiesterase type 5 inhibitors. Her ocular and auricular symptoms disappeared quickly. The hemodynamic parameters were also significantly improved after treatment. To our knowledge, this is the first RPC complicated with SSC-PAH reported.
\end{abstract}

Keywords

systemic sclerosis • pulmonary arterial hypertension • relapsing polychondritis

\section{Introduction}

Relapsing polychondritis (RPC) is an autoimmune disease characterized by recurrent and progressive cartilage inflammation. Patients with RPC are usually concurrent with autoimmune disorders, including rheumatic diseases, vasculitis, or malignancy. ${ }^{[1]}$ Systemic sclerosis (SSc) is a connective tissue disease (CTD) in which microvascular/endothelial damage, autoimmune response, and diffuse fibrosis are involved in the pathogenesis. Among SSc patients, those that developed pulmonary arterial hypertension (PAH) amounted to $7 \%-12 \% .^{[2]}$ There are very few reports about the concomitant RPC patients with SSc. ${ }^{[3]}$ Cases of RPC complicated with SSc-PAH are much fewer. A case of RPC complicated with SSc developed PAH is reported here.

Address for correspondence:

*Wei Wei, Department of Rheumatology and Immunology, Tianjin Medical University General Hospital, Tianjin Medical University, Tianjin 300052, China.

E-mail: tjweiwei316@163.com

Zhenwen Yang, Department of Cardiology, Tianjin Medical University General Hospital, Tianjin Medical University, Tianjin 300052, China.

E-mail: yzwmd@hotmail.com

\#Li Su and Na Zhang contributed equally to this study.

\section{Case presentation}

A 50-year-old woman who complained of edema and Raynaud's phenomenon for 3 months visited a local clinic in November 2016. The patient's skin was dark and tight, especially in her face, ears, hands, feet, bilateral forearms, and calves. Her anti-nuclear antibody (ANA), anti-U1 small nuclear ribonucleoprotein antibody, and anti-Ro/SSA antibody were positive. An echocardiogram was performed and showed right heart enlargement and PAH (estimated systolic pressure: $71 \mathrm{mmHg}$ ). No specific treatment was given at that time. In January 2018, her both auricles were swollen and painful with elevated local skin temperature, and yellow fluid was visible to the naked eye at the surface of her bilateral auricles. Her left auricle became soft gradually 

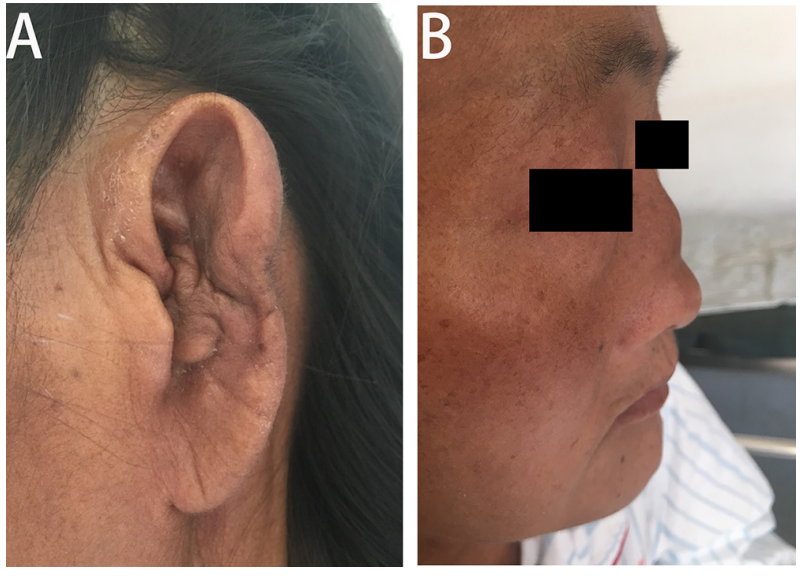

Figure 1: Clinical presentation. Cauliflower-like auricle (A) and saddle nose (B).

and evolved into a cauliflower-like appearance (Figure 1A). However, the swelling and pain disappeared spontaneously in May 2018. She came to us in June 2018 with red eyes and blurred vision. She was found to have iritis by an ophthalmologist, and saddle nose (Figure 1B). Laboratory data showed elevation of $\mathrm{N}$-terminal prohormone of brain natriuretic peptide (NT-proBNP), at $1336 \mathrm{pg} / \mathrm{mL}$, erythrocyte sedimentation rate (ESR), at $45 \mathrm{~mm} / \mathrm{h}$, and C-reactive protein (CRP) at $1.37 \mathrm{mg} / \mathrm{dL}$. Her ANA was positive with a titer of 1:800. Her blood anti-nRNP, anti-SSA, and anti-Ro-52 antibodies were positive, too. The complete blood count, renal, and liver function tests were all in the normal range. Furthermore, infectious and neoplastic diseases were excluded based on the related examinations.

Computed tomography angiography of the pulmonary artery showed no embolism but a dilated main pulmonary artery. Interstitial lung disease (ILD) was ruled out by chest high-resolution computed tomography. The echocardiography report revealed enlargement of the right heart (right atrium: $55 \mathrm{~mm}$; right ventricle: $45 \mathrm{~mm}$ ) and PAH (estimated systolic pressure: $103 \mathrm{mmHg}$ ). The thickness of the right ventricle wall increased to $8 \mathrm{~mm}$ and the tricuspid annulus plane systolic excursion was reduced to $13 \mathrm{~mm}$. The left ventricle was small $(33 \mathrm{~mm}$ ), while the left atrial dimension, left ventricular wall thickness, and motion were in the normal range. The left ventricular ejection fraction was $63 \%$. During right heart catheterization, her mean pulmonary arterial pressure (mPAP), pulmonary vascular resistance, and pulmonary artery wedge pressure were $57 \mathrm{mmHg}, 18$ Wood units, and $14 \mathrm{mmHg}$, respectively. Cardiac output $(\mathrm{CO})$, cardiac index $(\mathrm{Cl})$, and saturation of mixed venous blood oxygen (SvO2) were 2.3 $\mathrm{L} / \mathrm{min}, 1.6 \mathrm{~L} / \mathrm{min} / \mathrm{m}^{2}$, and $60 \%$, respectively. The diffusing capacity of the lung for carbon monoxide was $46.2 \%$ of the predicted value. The 6-min walk distance (6MWD)

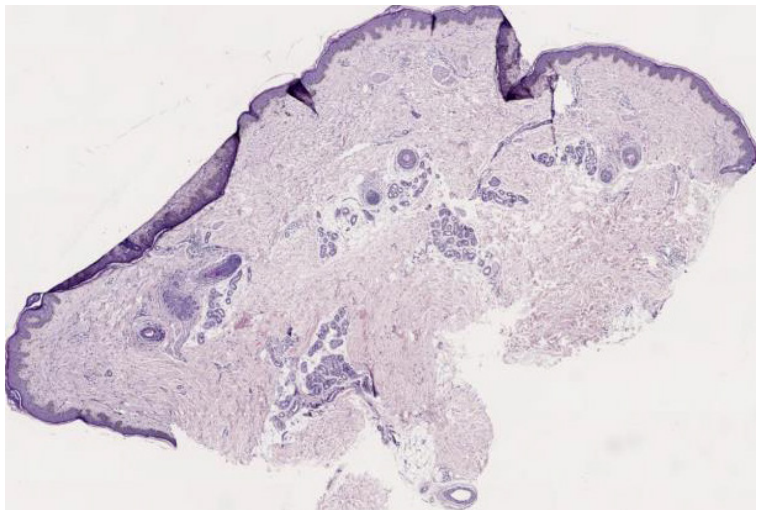

Figure 2: Histopathological findings. Skin biopsy showed increased pigmentation in the basal layer, mild inflammation around the superficial vascular layer of the dermis, and visible hyperplasia of the subcutaneous collagen fibers in the dermal melanocytes, which were all consistent with SSc.

was $354 \mathrm{~m}$. She had no symptoms suggesting laryngeal involvement and abnormalities of laryngeal or upper tracheal stenosis during physical examination. The histopathological findings of the skin biopsy were consistent with SSc (Figure 2).

SSc-PAH complicated with RPC was diagnosed. The patient was treated with methylprednisolone $40 \mathrm{mg}$ daily combined with oral cyclophosphamide (CYC) at a dose of $100 \mathrm{mg}$ QOD. Her ocular and auricular symptoms disappeared quickly. In addition, she received ambrisentan (5 mg daily) and sildenafil ( $75 \mathrm{mg}$ daily) treatment at the same time. The dose of methylprednisolone was gradually tapered to $4 \mathrm{mg}$ QD and maintained until March 2021. Her NT-proBNP, ESR, and CRP levels returned to normal range. Right heart catheterization was repeated and her mPAP decreased to $38 \mathrm{mmHg}$ in May 2020. CO, Cl, and SvO2 were increased to $4.5 \mathrm{~L} / \mathrm{min}, 2.9 \mathrm{~L} / \mathrm{min} / \mathrm{m}^{2}$, and $73 \%$, respectively. Moreover, her 6MWD was $426 \mathrm{~m}$. Repeated echocardiography revealed enlargement of the right heart (right atrium, $41 \mathrm{~mm}$; right ventricle, $36 \mathrm{~mm}$ ), and PAH (estimated systolic pressure: $47 \mathrm{mmHg}$ ).

\section{Discussion}

The patient presented with symmetrical auricular chondritis, saddle nose, and ocular inflammation, which meet the diagnosis of RPC according to the McAdam et al. ${ }^{[4]}$ criteria. The patient has also fulfilled the 2013 American College of Rheumatology/European League Against Rheumatism classification criteria for SSc. PAH was diagnosed by the results of right heart catheterization. In patients with RPC, 64\% presented complications with other autoimmune associated diseases, including vasculitis, systemic lupus erythematosus, and Sjögren's syndrome..$^{[5]}$ However, only one case of RPC-SSc was reported in the literature ${ }^{[3]}$ and this is the first 
RPC complicated with SSc-PAH reported according to our knowledge.

RPC is easy to be ignored when systemic diseases were diagnosed before RPC, because the symptoms of RPC, including fever, eye inflammation, and arthritis, can easily be explained by the concomitant rheumatic and infectious diseases. The previously reported case of RPC-SSc was diagnosed with SSc 5 years before RPC in the literature. ${ }^{[3]}$ In our case, timely diagnosis of RPC was not carried out until the auricle and nasal distortion happened. Since RPC is potentially fatal, early diagnosis and treatment may reduce morbidity and mortality. Since positive ANA in RPC is uncommon, when a significant high titer of ANA titer was found, concomitant rheumatic diseases should be considered. ${ }^{\left[{ }^{[6]}\right.}$ In addition, sufficient history-taking and physical examination can provide some clues for identifying the underlying systemic diseases.

The pathogenesis of RPC and SSc remains unclear. However, the shared HLA-DRB1 and HLA-DQB1 alleles might be the basis for the concurrent occurrence of RPC and SSc in a patient. ${ }^{[7,8]}$ Abnormal levels of cartilage oligomeric matrix protein and soluble triggering receptor expressed on myeloid cells-1 were detected in both RPC and SSc, which could be the serological markers for disease activity. Vascular involvement in RPC was in the range of $5 \%-25 \%$. ${ }^{[9]}$ However, the role of RPC in PAH is still unclear and needs further research.

The treatment for patients with SSc and RPC are corticosteroids and immunosuppressive drugs. However, although corticosteroids are commonly used for RPC treatment, they are a risk factor for scleroderma renal crisis. We should avoid unnecessary and long-term use of corticosteroids when treating patients with SSc complicated with RPC. CYC may be effective in relieving skin changes of patients with $\mathrm{SSc}^{[10]}$ and it has also been shown to be effective in treating RPC. Therefore, CYC should be given to patients with SSc and RPC. PAH is refractory to corticosteroids or immunosuppressive drugs, ${ }^{[11]}$ and double- or triple-combination therapies (e.g., oral endothelin-A receptor antagonist and phosphodiesterase type 5 inhibitors) were recommended. In this case, the patient's hemodynamic parameters were significantly improved after treatment with ambrisentan and sildenafil.

Conflict of Interest

The authors state that they have no conflict of interest.

Patient Informed Consent Declaration

The authors certify that they have obtained all appropriate patient consent documents. In the documents, the patient has given her consent for her images and other clinical information to be published in the journal.

\section{References}

[1] Borgia F, Giuffrida R, Guarneri F, et al. Relapsing Polychondritis: An Updated Review. Biomedicines. 2018;6(3):84.

[2] Hickey PM, Lawrie A, Condliffe R. Circulating Protein Biomarkers in Systemic Sclerosis Related Pulmonary Arterial Hypertension: A Review of Published Data. Front Med (Lausanne). 2018;5:175.

[3] Sugisaki K, Takeda I, Kanno T, et al. A Case Report of Relapsing Polychondritis with an Auricular Ulcer Complicated by Systemic Sclerosis. Ryumachi. 2002;42(3):610-617.[in Japanese].

[4] McAdam LP, O'Hanlan MA, Bluestone R, et al. Relapsing polychondritis: prospective study of 23 patients and a review of the literature. Medicine (Baltimore). 1976 May;55(3):193-215.

[5] Ghib LJ, Damian L, Andrei M, et al. THU0470 Association of Relapsing Polychondritis with Other Autoimmune Conditions: Experience of a Romanian Center. Ann Rheum Dis. 2013;72:A323.

[6] Piette JC, El-Rassi R, Amoura Z. Antinuclear Antibodies in Relapsing Polychondritis. Ann Rheum Dis. 1999;58(10):656-657.
[7] Gourh P, Safran SA, Alexander T, et al. HLA and Autoantibodies Define Scleroderma Subtypes and Risk in African and European Americans and Suggest a Role for Molecular Mimicry. Proc Natl Acad Sci U S A. 2020;117(1):552-562.

[8] Terao C, Yoshifuji H, Yamano Y, et al. Genotyping of Relapsing Polychondritis Identified Novel Susceptibility HLA Alleles and Distinct Genetic Characteristics from Other Rheumatic Diseases. Rheumatology (Oxford). 2016;55(9):1686-1692.

[9] D'Cruz DP, Ferrada MA. Relapsing Polychondritis and Largevessel Vasculitis. J Rheumatol. 2020;47(12):1732-1733.

[10] Kersten BE, den Broeder $\mathrm{N}$, van den Hoogen $\mathrm{FHJ}$, et al. Treatment with Cyclophosphamide i.v. Pulse Therapy is an Option for Effective Treatment of Skin Fibrosis in Patients with Early Systemic Sclerosis. Rheumatology (Oxford). 2020;59(7):1550-1555.

[11] Almaaitah S, Highland KB, Tonelli AR. Management of Pulmonary Arterial Hypertension in Patients with Systemic Sclerosis. Integr Blood Press Control. 2020;13:15-29. 